\title{
Ability of Latin America Laboratories to Detect Antimicrobial Resistance Patterns: Experience of the SENTRY Antimicrobial Surveillance Program (1997-2000)
}

\author{
Rodrigo E. Mendes ${ }^{1}$, Adriana O. Reis ${ }^{1}$, Ana C. Gales ${ }^{1}$, \\ Ronald N. Jones ${ }^{2}$ and Hélio S. Sader ${ }^{1,2}$
}

Special Laboratory of Clinical Microbiology, Federal University of São Paulo, SP, Brazill'; The Jones Group/ Jones Microbiology Institute Laboratories, North Liberty, IA, USA ${ }^{2}$.

The accuracy of antimicrobial susceptibility tests is a crucial step for the clinical management of patients with serious infections. They must be reliable and precise because they will guide antimicrobial therapy. Our main objective was to compare the results of susceptibility testing performed by the SENTRY coordinator laboratory with those reported by the participating Latin American medical centers. A total of 10,277 bacterial isolates were tested by the reference broth microdilution method at the coordinator laboratory in the United States. The tests were performed and interpreted following the National Committee for Clinical Laboratory Standards (NCCLS) recommendations. Ten antimicrobial agent-organism combinations were analyzed. The susceptibility methods utilized in each of the medical centers were also evaluated. Total agreement of the results was obtained in nearly $88 \%$ of the antimicrobial agent-organism combinations. "Very major" (false-susceptible results) and "major errors" (false-resistant results) were observed in $12 \%$ and $6 \%$ of the cases, respectively. The highest disagreements were observed for coagulasenegative Staphylococcus - oxacillin (20\% - very major error) and Burkholderia cepacia -imipenem (21\% - very major error). The susceptibility method with the highest agreement rate was Etest $B$ $(92 \%)>$ PASCO® $(91 \%)>$ agar dilution $(91 \%)>$ MicroScan $®(90 \%)>$ Vitek® $(87 \%)$. External quality assurance data obtained by surveillance programs such as the SENTRY Antimicrobial Surveillance Program are not only helpful for detecting the emergence of patterns of antimicrobial resistance, but also to monitor the performance of the participating microbiology laboratories.

Key Words: Antimicrobial susceptibility test, antimicrobial resistance pattern, quality assurance.

The clinical microbiology laboratory plays an important role in antibiotic selection and in the performance of routine antimicrobial susceptibility testing of clinical isolates [1]. Antimicrobial resistance has emerged in bacterial pathogens throughout the

Received on 15 March 2003; revised 09 October 2003.

Address for correspondence: Dr. Rodrigo Elisandro Mendes, M.Sc. Special Clinical Microbiology Laboratory, Division of Infectious Diseases, Federal University of São Paulo. Rua Leandro Dupret, 188, São Paulo, SP, Brazil, Zip code: 04025010. Phone/Fax: (55-11) 5081-2819/5571-5180/5081-2965

E-mail: lemcdipa@terra.com.br.

Homepage: http://www.unifesp.br/dmed/dipa/lemc/

The Brazilian Journal of Infectious Diseases 2003;7(5):282-289 (C) 2003 by The Brazilian Journal of Infectious Diseases and Contexto Publishing. All rights reserved. world [2], and the Enterobacteriaceae, Pseudomonas spp., Staphylococcus spp. and Enterococcus spp. are just a few examples of the bacteria involved in the rising problem of resistance detected by both national and international surveillance programs during the past few years [3-6]. For this reason, antimicrobial susceptibility testing has become an essential component in guiding antimicrobial therapy.

Nosocomial infections are the main cause of morbidity and mortality in the Unites States. Hospitalized patients are more susceptible to infections because of their underlying disease conditions, in addition to exposure during invasive procedures [7]. Microorganisms isolated from these patients may be resistant, and it can be difficult to detect emerging 
antimicrobial resistance patterns and to ensure the efficiency of reporting strategies for antimicrobial resistance. Failure of a test to predict antimicrobial resistance can result in increased morbidity or mortality. A wide variety of antimicrobial susceptibility systems are available to clinical microbiology laboratories. They must be reliable and precise, because their results will guide antimicrobial therapy. The main objective of our study was to compare the susceptibility testing results of the reference methodology performed by the SENTRY coordinator laboratory with those reported by the Latin American participating medical centers in order to assess the accuracy of the data submitted by the participating laboratories.

\section{Materials and Methods}

Study design. The SENTRY Antimicrobial Surveillance Program was established to monitor the prominent pathogens and antimicrobial resistance patterns of nosocomial and community-acquired infections via a broad network of sentinel hospitals distributed by geographic location andsize. The monitored infections include blood stream infections (BSI), outpatient respiratory infections, lower respiratory tract infections in hospitalized patients (LRTI), skin and soft tissue infectionsin hospitalized patients (SSTI), and urinary tract infections in hospitalized patients (UTI). The participating laboratories were distributed throughout seven countries, including Brazil (São Paulo, Rio de Janeiro, Porto Alegre and Florianopolis), Argentina (Buenos Aires and San Isidro), Chile (Santiago, two centers), Colombia (Medellin), Mexico (Mexico City, three centers) and Uruguay (Montevideo). In 1998, the center located in Montevideo was replaced by a Venezuelan center located in Caracas.

Each participating center contributed results (organismidentification, date of isolation, antimicrobial susceptibility profile, etc) for the first 20 consecutive episodes of BSI per month, 100 consecutive episodes of LRTI per year, 50 consecutive isolates from SSTI peryear, and 50 consecutiveisolates from UTI per year. Only one isolate per patient was included in the study and allisolates were saved onagar slants and sent to the coordinating laboratory (RN Jones, Iowa, USA) for storage and further characterization by reference identification and susceptibility testing methods. All isolates recovered and data collected from January 1997 to December 2000 were evaluated.

Species identification. All isolates were identified at the participating institution by the routine methodology in use in each laboratory. On receipt at the University of Iowa, isolates were subcultured on bloodagar to ensure viability and purity. Species identifications were confirmed or performed with the use of the Vitek ${ }^{\circledR}$ system (bioMérieux Vitek ${ }^{\circledR}$ ) or API (bioMérieux Vitek $\left.{ }^{\circledR}\right)$ products, along with standard reference methods. Isolates were frozen at $-70^{\circ} \mathrm{C}$ until they were processed.

Susceptibility testing. Antimicrobial susceptibility testing of isolates was performed at the reference laboratory by reference broth microdilution methods as described by the National Committee for Clinical Laboratory Standard (NCCLS) [8]. Microdilution trays were purchased from MicroScan $\AA$, TREK, and PML Microbiologicals. Antimicrobial agents were obtained from their respective manufacturers.

Quality control. Quality control was performed at the coordinator laboratory by testing E. coli American Type Culture Collection (ATCC) 25922, Staphylococcus aureus ATCC 29213, Pseudomonas aeruginosa ATCC 27853, Streptococcus pneumoniae ATCC 49619, and Enterococcus faecalis ATCC 29212. Interpretive criteria for each antimicrobial tested were those published by the NCCLS.

Categorical agreement. Broth microdilution performed at the coordinating laboratory was considered the reference method (gold standard). Broth microdilution results were compared to the antimicrobial susceptibility results submitted by the participating medical laboratories using the local routine tests, including: disk diffusion, agar-dilution, MicroScan ${ }^{\circledR}$, Vitek ${ }^{\circledR}$, Pasco $\AA$ and Etest ${ }^{\circledR}$. Categorical agreement was considered when the test result provided by the 
participating laboratory and the result of the reference laboratory was in the same susceptibility category. Susceptibility categories were determined according to the NCCLS breakpoint recommendations [8]. A previous NCCLS document [9] was used for coagulase-negative Staphylococcus isolates in 1997 and 1998, since the oxacillin breakpoint for this microorganism had changed in 1999.

Errors were determined by previously published methods. "Very major" errors indicate false-susceptible results and they were calculated by dividing the number of false-susceptible results reported by the participating centers by the number of true resistant strains found by the reference laboratory. "Major" errors indicate falseresistant results and they were calculated by dividing the number of false-resistant results reported by the participating centers by the number of true resistant strains based found by the reference laboratory. Rates of category agreement $<10.0 \%$, very major $<1.5 \%$ and major error $<5.0 \%$ were considered acceptable [10].

Ten antimicrobial agent-organism combinations were studied for all sites: Staphylococcus aureusoxacillin; coagulase-negative Staphylococcus (CoNS)-oxacillin; Klebsiella spp.-ceftriaxone; Klebsiella spp.-ceftazidime; $E$. coli-ceftriaxone; $E$. coli-ceftazidime; Acinetobacter spp.-imipenem; Burholderia cepacia-imipenem; Stenotrophomonas maltophilia-imipenem; and Pseudomonas aeruginosa-imipenem. The antimicrobial agentorganism CoNS-oxacillin analysis combinations were divided into two different steps since the oxacillin breakpoint had been modified during the study interval. SPSS for Windows Release 10.0.5 Standard Version was used to perform statistical analyses.

\section{Results}

The antimicrobial susceptibility testing methods used by the participating medical centers, and the respective number of isolates tested in the SENTRY Program, are shown in Table 1. The susceptibility test methods were discriminated by the participating medical center for only 4,159 (40.5\%) isolates. The most frequent method used by the participating laboratories was disk diffusion $(23.4 \%)$, followed by the automated Vitek ${ }^{\circledR}$ system $(9.2 \%)$.

Overall, the rate of categorical agreement was $88.5 \%$. The combination B. cepacia-imipenem showed the lowest categorical agreement $(51.7 \%)$, followed by Klebsiella spp.- ceftriaxone (77.9\%), Klebsiella spp.- ceftazidime (79.4\%), and CoNSoxacillin $(79.6 \%)$. The other antimicrobial agent organism combinations showed $>90 \%$ categorical agreements (Table 2).

The "very major" errors ranged from $0.0 \%$ for $E$. coli-ceftriaxone to $20.5 \%$ for CoNS-oxacillin; with the exception of the former combination, none of the antimicrobial agent-organism combinations presented an acceptable rate of very major errors. When "major" errors were analyzed, only $60 \%$ of the antimicrobial agent-organism combinations showed acceptable rates $(\leq 5.0 \%)$.

To evaluate the susceptibility test systems, it was necessary to determine the power of discrimination of the various antimicrobial susceptibility test methodologies used by the participating centers, followed by the respective susceptibility test results. Only 3,268 isolates had the susceptibility tests systems data accompanied by the corresponding susceptibility tests results. Etest ${ }^{\circledR}$, PASCO $®$, agar dilution and MicroScan $\AA$ showed the highest categorical agreement $(92.6 \%, 91.6 \%, 91.3 \%$ and $90.1 \%$, respectively). The percentage of "very major" error varied from $5.2 \%$ for agar dilution to $25.0 \%$ for the Etest ${ }^{\circledR}$ (Table 3).

The categorical agreement varied from $83.6 \%$ for medical center 092 to $100.0 \%$ for medical center 042 . Except for medical center 042, none of the centers had acceptable "very major" error rates (Table 4).

\section{Discussion}

Similar to what occurs in Latin American routine microbiology laboratories, the Kirby-Bauer disk diffusion test was the most common (23.5\%) antimicrobial susceptibility test used by the Latin American medical centers participating in this study. The predominance of disk diffusion is probably due to 
Table 1. Antimicrobial susceptibility testing methods used and the respective number of isolates tested by each of the participating medical centers in the SENTRY Antimicrobial Surveillance Program

\begin{tabular}{|c|c|c|c|c|c|c|c|c|c|}
\hline \multirow{2}{*}{$\begin{array}{l}\text { Medical } \\
\text { Center }\end{array}$} & \multicolumn{6}{|c|}{ No. of isolates tested by each methodology } & \multirow[t]{2}{*}{ Total } & \multirow[t]{2}{*}{ Not available $^{\text {b }}$} & \multirow[t]{2}{*}{ Total } \\
\hline & Agar dilution & Disk diffusion & Etest $\AA^{a}$ & MicroScana & Pasco $^{a}$ & Vitek $^{\mathbf{a}}$ & & & \\
\hline 039 & 151 & 203 & & & & 25 & 379 & 671 & 1,050 \\
\hline 040 & & 162 & & & 49 & & 211 & 898 & 1,109 \\
\hline 041 & & 17 & & & & 239 & 256 & 248 & 504 \\
\hline 042 & 01 & & & & & & 01 & 976 & 977 \\
\hline 043 & 124 & 39 & & & & 81 & 244 & 887 & 1,131 \\
\hline 044 & & 51 & & 288 & & & 339 & 552 & 891 \\
\hline 045 & & 05 & & 96 & & 142 & 243 & 314 & 557 \\
\hline 046 & & 112 & & & & 443 & 555 & 574 & 1,129 \\
\hline 047 & & 87 & 40 & & & & 127 & 58 & 185 \\
\hline 048 & & 1,416 & & & & & 1416 & 21 & 1,437 \\
\hline 049 & & 71 & & & & & 71 & 399 & 470 \\
\hline 056 & & & & & & 15 & 15 & 21 & 36 \\
\hline 057 & & 242 & & & & & 242 & 427 & 669 \\
\hline 092 & 60 & & & & & & 60 & 72 & 132 \\
\hline Total & $\begin{array}{c}336 \\
(3.3 \%)\end{array}$ & $\begin{array}{c}2,405 \\
(23.4 \%)\end{array}$ & $\begin{array}{c}40 \\
(0.4 \%)\end{array}$ & $\begin{array}{c}384 \\
(3.7 \%)\end{array}$ & $\begin{array}{c}49 \\
(0.5 \%)\end{array}$ & $\begin{array}{c}945 \\
(9.2 \%)\end{array}$ & $\begin{array}{c}4,159 \\
(40.5 \%)\end{array}$ & $\begin{array}{c}6,118 \\
(59.5 \%)\end{array}$ & 10,277 \\
\hline
\end{tabular}

a-Etest ${ }^{\circledR}$ (AB Biodisk, Solna, Sweden); MicroScan ${ }^{\circledR}$ (Dade Behring, West Sacramento, California); Vitek® (bioMérieux, Hazelwood); PASCO (Becton-Dickinson, Wheatridge, Colombia). b - Methodology not reported. 
Table 2. Accuracy data of antimicrobial agent-organism combinations

\begin{tabular}{|c|c|c|c|c|c|}
\hline \multirow[t]{2}{*}{ Microorganism } & \multirow{2}{*}{$\begin{array}{l}\text { Antimicrobial } \\
\text { agents }\end{array}$} & \multirow[t]{2}{*}{ Number tested } & \multirow{2}{*}{$\begin{array}{c}\text { \% Categorical } \\
\text { agreement }^{\mathrm{a}}\end{array}$} & \multicolumn{2}{|c|}{ \% Error (errors/available results) } \\
\hline & & & & Very major $^{b}$ & Major $^{c}$ \\
\hline B. cepacia & Imipenem & 58 & 51.7 & $21.1(4 / 19)$ & $25.0(7 / 28)$ \\
\hline $\mathrm{CoNS}^{\mathrm{d}}$ & Oxacillin & 986 & 79.6 & $20.5(146 / 710)$ & $17.7(49 / 276)$ \\
\hline \multirow[t]{2}{*}{ Klebsiella spp. } & Ceftriaxone & 249 & 77.9 & $13.2(7 / 53)$ & $11.0(19 / 173)$ \\
\hline & Ceftazidime & 776 & 79.4 & $5.8(17 / 295)$ & $20.4(89 / 436)$ \\
\hline P. aeruginosa & Imipenem & 1274 & 86.9 & $12.8(30 / 233)$ & $3.8(37 / 955)$ \\
\hline \multirow[t]{2}{*}{ Escherichia coli } & Ceftriaxone & 441 & 93.6 & $0.0(0 / 10)$ & $3.1(13 / 415)$ \\
\hline & Ceftazidime & 1065 & 91.4 & $19.7(13 / 66)$ & $5.0(49 / 974)$ \\
\hline S. malthophilia & Imipenem & 82 & 91.4 & $8.6(7 / 81)$ & $0.0(0 / 1)$ \\
\hline Acinetobacter spp. & Imipenem & 545 & 92.1 & $12.1(8 / 66)$ & $4.4(21 / 471)$ \\
\hline S. aureus & Oxacillin & 2278 & 95.1 & $7.7(59 / 764)$ & $3.4(52 / 1514)$ \\
\hline Total & & 7754 & 88.5 & $12.7(291 / 2297)$ & $6.4(336 / 5243)$ \\
\hline
\end{tabular}

a - Number of tests with category agreement divided by the number of organisms tested.

$\mathrm{b}-\%$ very major error: number of false-susceptible strains divided by the number of true resistant strains.

$\mathrm{c}-\%$ major error: number of false-resistant strains divided by the number of true susceptible strains.

d - Coagulase-negative Staphylococcus.

Table 3. Evaluation of the antimicrobial susceptibility test systems used by the Latin American medical centers

\begin{tabular}{|c|c|c|c|c|}
\hline \multirow[t]{2}{*}{ Methodology ${ }^{\mathrm{a}}$} & \multirow{2}{*}{$\begin{array}{c}\text { Number of } \\
\text { isolates tested }\end{array}$} & \multirow{2}{*}{$\begin{array}{c}\% \text { Categorical }^{\mathrm{b}} \\
\text { agreement }^{-}\end{array}$} & \multicolumn{2}{|c|}{ \% Error (errors/available results) } \\
\hline & & & Very major ${ }^{c}$ & Major $^{d}$ \\
\hline Etest & 27 & 92.6 & $25.0(1 / 4)$ & $4.3(1 / 23)$ \\
\hline PASCO & 36 & 91.6 & $0.0(0 / 2)$ & $3.1(1 / 32)$ \\
\hline Agar dilution & 276 & 91.3 & $5.2(5 / 96)$ & $7.4(13 / 176)$ \\
\hline MicroScan & 314 & 90.1 & $12.3(7 / 57)$ & $7.1(18 / 252)$ \\
\hline Vitek & 682 & 89.7 & $11.0(18 / 163)$ & $6.1(31 / 502)$ \\
\hline Disk-diffusion & 1933 & 87.3 & $11.5(83 / 720)$ & $5.9(68 / 1147)$ \\
\hline
\end{tabular}

a-Etest ${ }^{\circledR}\left(\right.$ AB Biodisk, Solna, Sweden); MicroScan ${ }^{\circledR}$ (Dade Behring, West Sacramento, California); Vitek $®$ (bioMérieux, Hazelwood); PASCO (Becton-Dickinson, Wheatridge, Colombia).

$\mathrm{b}$ - Number of tests with category agreement divided by the number of organisms tested.

c $-\%$ very major error: number of false-susceptible strains divided by the number of true resistant strains.

$\mathrm{d}-\%$ major error: number of false-resistant strains divided by the number of true susceptible strains. 
Table 4. Evaluation of susceptibility test accuracy according in each of the medical centers

\begin{tabular}{|c|c|c|c|c|}
\hline \multirow[t]{2}{*}{ Medical center } & \multirow{2}{*}{$\begin{array}{c}\text { Number of } \\
\text { isolates tested }\end{array}$} & \multirow{2}{*}{$\begin{array}{c}\% \text { Categorical } \\
\text { agreement }^{\mathrm{a}}\end{array}$} & \multicolumn{2}{|c|}{ \%Error (errors/available results) } \\
\hline & & & Very major & Major $^{c}$ \\
\hline 039 & 867 & 90.9 & $5.1(13 / 256)$ & $6.3(37 / 587)$ \\
\hline 040 & 964 & 86.1 & $8.4(28 / 334)$ & $10.7(63 / 590)$ \\
\hline 041 & 419 & 89.5 & $9.3(10 / 107)$ & $4.7(14 / 296)$ \\
\hline 042 & 17 & 100.0 & $0.0(0 / 11)$ & $0.0(0 / 6)$ \\
\hline 043 & 724 & 86.6 & $22.3(68 / 305)$ & $5.3(22 / 413)$ \\
\hline 044 & 903 & 90.0 & $13.6(17 / 125)$ & $5.7(43 / 752)$ \\
\hline 045 & 409 & 88.5 & $35.4(23 / 65)$ & $3.6(12 / 332)$ \\
\hline 046 & 924 & 92.4 & $12.2(25 / 205)$ & $4.1(29 / 705)$ \\
\hline 047 & 152 & 94.7 & $13.0(3 / 23)$ & $1.6(2 / 125)$ \\
\hline 048 & 1189 & 86.0 & $13.6(71 / 522)$ & $7.2(46 / 634)$ \\
\hline 049 & 409 & 82.4 & $6.0(7 / 116)$ & $16.3(45 / 276)$ \\
\hline 056 & 38 & 92.1 & $15.4(2 / 13)$ & $4.0(1 / 25)$ \\
\hline 057 & 643 & 90.5 & $11.8(22 / 187)$ & 3.7 16/431) \\
\hline 092 & 49 & 83.6 & $10.0(2 / 20)$ & $16.6(4 / 24)$ \\
\hline
\end{tabular}

a - Number of tests with category agreement divided by the number of organisms tested.

$\mathrm{b}-\%$ very major error: number of false-susceptible strains divided by the number of true resistant strains.

$\mathrm{c}-\%$ major error: number of false-resistant strains divided by the number of true susceptible strains.

low cost, flexibility and demonstrated accuracy. However, the use of semi-quantitative automated systems has recently been increasing [11].

Detection of methicillin-resistant coagulase-negative Staphylococcus spp. isolates by phenotypic methods is a challenge for most clinical microbiology laboratories. The NCCLS recently proposed that the most sensitive, practical method for the detection of this type of resistance was either the broth microdilution determination of the oxacillin MIC or the oxacillin disk diffusion test, with breakpoints specific for coagulasenegative Staphylococcus spp. [12]. However, the participating laboratories showed a high rate of errors for the CoNS-oxacillin combination $(20.5 \%)$. This result leads us to suggest that the participating centers may not be applying the latest NCCLS document criteria for interpretation.

High rates of false-susceptibility results ("very major" errors) were encountered among Klebsiella spp. strains when the pathogen was tested against ceftriaxone (13.2\%) and ceftazidime $(5.8 \%)$. The $E$. coli-ceftazidime combination also showed high rates of "very major" errors (19.7\%). The high rates of errors described above may be explained by the high prevalence of extended-spectrum $\beta$-lactamase (ESBL) producing strains among these species throughout Latin America. Because of differences in drug substrate affinities of some ESBLs, and the resulting inoculum effects with standard susceptibility tests, ESBL producing isolates may give rise to inconsistent results when tested by any methodology [13].

Acinetobacter spp. and $P$. aeruginosa are highly prevalent pathogens in Latin America. In addition, the nosocomial prevalence of S. maltophilia is increasing. When these pathogens were tested against imipenem we expected high rates of false-resistant results (major errors), since the instability of imipenem could cause these types of errors. However, among these species, the major errors were within acceptable rate levels $(<5.0 \%)$. On the other hand, high frequencies of false- 
susceptibility results (very major errors) from $8.6 \%$ to $12.8 \%$ were observed among these non-fermenting pathogens.

Burholderia cepacia strains showed the lowest categorical agreement $(51.7 \%)$ and the highest rates of very major $(21.1 \%)$ and major errors $(25.0 \%)$. Additionally, although $S$. malthophilia is intrinsically resistant to imipenem, several of the participating laboratories reported seven isolates as susceptible. These poor results are probably due to the lack of standardized susceptibility testing methods for both $S$. malthophilia and B. cepacia.

Stenotrophomonas maltophilia and B. cepacia isolates present problems since the interpretative category results of disk diffusion and automated systems may not agree with susceptibility categories based on reference MIC determination. Pankuch et al. [14] also found a poor correlation among five susceptibility testing methods evaluated against eight $\beta$-lactams (including $\beta$-lactam- $\beta$-lactamase inhibitor combinations) and ciprofloxacin antimicrobial agents against $S$. maltophilia.

The NCCLS currently recommends that only reference quantitative testing methods should be used on S. maltophilia [8]. Unfortunately, these methods are labor-intensive, and thus, difficult to incorporate into routine laboratory procedures. Susceptibility testing of S. maltophilia by disk diffusion or by semiautomated methods, such as Vitek (bioMerieux Inc., Hazelwood, MO.) or MicroScan (Baxter Health Care Corp., Sacramento, CA) is unreliable and therefore not recommended [15].

In the evaluation of the susceptibility test methods/ systems, "very major" error rates varied from 5.2\% to $25.0 \%$ depending on the methodology utilized and the species tested (Table 3). The limited number of truely resistant isolates may have influenced the major error rates of some methodologies. Only 57 and 4 true resistant isolates were tested by MicroScan ${ }^{\circledR}$ and Etest ${ }^{\circledR}$. Thus, a low number of false susceptible reports could result in unacceptable rates of very major errors.

In the $S$. aureus-oxacillin combination, among 7 truly resistant strains, 2 isolates (29\%) were reported as susceptible by MicroScan ${ }^{\circledR}$ (data not shown).
Automated systems have excellent specificity but often do not have sensitivity in detecting methicillin-resistant staphylococci, probably due to the reduced incubation period (24 hours) and lack of $2 \% \mathrm{NaCl}$ [16]. A similar problem was observed when using the disk diffusion test method, where 49 out of 83 "very major" errors were detected in the CoNS-oxacillin combination. Additionally, among the Vitek ${ }^{\circledR}$ system results the highest rate of "very major" errors was detected for the $P$. aeruginosa-imipenem combination $(8 / 18,44 \%)$. This result was unexpected since false-resistant errors would be prominent, as described above.

Except for the "major error" rates described for medical centers 043 and 045, categorical agreement and "very major" errors occurred at similar rates in all participating medical centers. The high "very major" rates observed in medical centers 043 and 045 were caused by the CoNS-oxacillin combination. These results could also be explained by the use of the previously used oxacillin breakpoint [9].

Generally speaking, we found low rates of categorical agreement, and high rates of "very major" and "major" errors. The fact that only problematic antimicrobial agents-organism combinations were selected for evaluation may have resulted in an overestimate of the error rates. In addition, clinical laboratories should not rely on a single susceptibility testing methodology to detect emerging resistant organisms [17].

It is important to recognize that subtle resistance mechanisms, especially among fastidious and slowgrowing organisms, may not be detected by any methodology with the same level of reproducibility and accuracy that can be expected for most other organismantibiotic combinations. Additionally, it is possible to perform a test correctly but report inaccurate results, particularly if obsolete guidelines or criteria are used. The correct interpretation and reporting of the antimicrobial susceptibility test results is as important as the ability to detect the resistance mechanism accurately.

External quality assurance data obtained by surveillance programs such as the SENTRY Antimicrobial Surveillance Program are not only helpful 
for detecting the emergence of patterns of antimicrobial resistance, but also for monitoring the performance of the participating microbiology laboratories.

\section{References}

1. Jorgensen J.H. Selection criteria for an antimicrobial susceptibility testing system. J Clin Microbiol 1993;31:2841-4.

2. Diekema D.J., Pfaller M.A., Jones R.N., et al. Trends in antimicrobial susceptibility of bacterial pathogens isolated from patients with bloodstream infections in the USA, Canada and Latin America. SENTRY Participants Group. Int J Antimicrob Agents 2000;13:257-71.

3. Diekema D.J., Pfaller M.A., Jones R.N., et al. Trends in antimicrobial susceptibility of bacterial pathogens isolated from patients with bloodstream infections in the USA, Canada and Latin America. SENTRY Participants Group. Int J Antimicrob Agents 2000;13:257-71.

4. Diekema D.J., Pfaller M.A., Schmitz F.J., et al. Survey of infections due to Staphylococcus species: Frequency of occurrence and antimicrobial susceptibility of isolates collected in the United States, Canada, Latin America, Europe, and the Western Pacific region for the SENTRY Antimicrobial Surveillance Program, 1997-1999. Clin Infect Dis 2001;32:114-32.

5. Gales A.C., Jones R.N., Forward K.R., et al. Emerging importance of multidrug-resistant Acinetobacter species and Stenotrophomonas maltophilia as pathogens in seriously ill patients: Geographic patterns, epidemiological features, and trends in the SENTRY Antimicrobial Surveillance Program (1997-1999). Clin Infect Dis 2001;32:104-13.

6. Sader H.S., Gales A.C., Pfaller M.A.,et al. Pathogen frequency and resistance patterns in Brazilian hospitals: Summary of results from three years of the SENTRY Antimicrobial Surveillance Program. Braz J Infect Dis 2001;5:200-14.

7. Emori T.G., Gaynes R.P. An overview of nosocomial infections, including the role of the microbiology laboratory. Clin Microbiol Rev 1993;6:428-42.

8. National Committee for Clinical Laboratory Standards. Methods for dilution antimicrobial susceptibility tests for bacteria that grow aerobically. Approved standard M7-A5. Wayne, Pennsylvania: 2000.

9. National Committee for Clinical Laboratory Standards. Methods for dilution antimicrobial susceptibility tests for bacteria that grow aerobically. Approved standard M7-A5. Wayne, Pennsylvania: 1998.
10. Isenberg H.D., Mulder R.H., Farnham S.M., Grinius B. Evaluating antimicrobial susceptibility systems. In: Isenberg H.D., ed. Clinical Microbiology Procedures Handbook. Washington, DC.: American Society for Microbiology, 1992.

11. Kiehlbauch J.A., Hannett G.E., Salfinger M., et al. Use of the National Committee for Clinical Laboratory Standards guidelines for disk diffusion susceptibility testing in New York state laboratories. J Clin Microbiol 2000;38:3341-8.

12. Jorgensen J.H., Ferraro M.J. Antimicrobial susceptibility testing: General principles and contemporary practices. Clin Infect Dis 1998;26:973-80.

13. Bush K. New beta-lactamases in gram-negative bacteria: Diversity and impact on the selection of antimicrobial therapy. Clin Infect Dis 2001;32:1085-9.

14. Pankuch G.A., Jacobs M.R., Rittenhouse S.F., Appelbaum P.C. Susceptibilities of 123 strains of Xanthomonas maltophilia to eight beta-lactams (including betalactam-beta-lactamase inhibitor combinations) and ciprofloxacin tested by five methods. Antimicrob Agents Chemother 1994;38:2317-22.

15. Yao J.D., Louie M., Louie L., et al. Comparison of E test and agar dilution for antimicrobial susceptibility testing of Stenotrophomonas (Xanthomonas) maltophilia. J Clin Microbiol 1995;33:1428-30.

16. Chambers H.F. Methicillin resistance in staphylococci: Molecular and biochemical basis and clinical implications. Clin Microbiol Rev 1997;10:781-91.

17. Winokur P.L., Canton R., Casellas J.M., Legakis N. Variations in the prevalence of strains expressing an extended-spectrum beta-lactamase phenotype and characterization of isolates from Europe, the Americas, and the Western Pacific region. Clin Infect Dis 2001;32:94-103. 